Check for updates

Cite this: Soft Matter, 2019, 15,6006

Received 16th April 2019, Accepted 28th June 2019 DOI: $10.1039 / \mathrm{c} 9 \mathrm{sm} 00772 \mathrm{e}$ rsc.li/soft-matter-journal

\title{
Bending rigidity of charged lipid bilayer membranes
}

\author{
Hammad A. Faizi, ${ }^{\text {ab }}$ Shelli L. Frey, ${ }^{\text {bc }}$ Jan Steinkühler, ${ }^{b}$ Rumiana Dimova (D) *b and \\ Petia M. Vlahovska id *d
}

\begin{abstract}
We experimentally investigate the effect of lipid charge on the stiffness of bilayer membranes. The bending rigidity of membranes with composition 0-100 mol\% of charged lipids, in the absence and presence of salt at different concentrations, is measured with the flicker spectroscopy method, using the shape fluctuations of giant unilamellar vesicles. The analysis considers both the mean squared amplitudes and the time autocorrelations of the shape modes. Our results show that membrane charge increases the bending rigidity relative to the charge-free membrane. The effect is diminished by the addition of monovalent salt to the suspending solutions. The trend shown by the membrane bending rigidity correlates with zeta potential measurements, confirming charge screening at different salt concentrations. The experimental results in the presence of salt are in good agreement with existing theories of membrane stiffening by surface charge.
\end{abstract}

\section{Introduction}

Cells and internal cellular organelles are enveloped by membranes composed primarily of lipid bilayers. Many cell biological functions involve membrane structural and morphological changes. ${ }^{1-3}$ These processes depend strongly on the mechanical properties of the lipid bilayer. A key characteristic that has been extensively studied is the bending rigidity, ${ }^{4}$ which quantifies the energy needed to change the membrane curvature. In neutral (charge-free) membranes, the bending rigidity is related to the energy cost for compression and expansion of the inner and outer lipid monolayers in the bent bilayer. Since biological membranes contain charged lipids, e.g., the fraction of phosphatidylglycerol in bacteria Staphylococcus aureus and Caulobacter crescentus can be as high as $80 \%,{ }^{5-8}$ an important question is how the presence of surface charge affects the bending rigidity of lipid bilayer membranes.

Surface charge is expected to stiffen the membrane because the repulsion between the charged lipids effectively resists membrane bending. This effect has been extensively studied theoretically within the framework of the Poisson-Boltzmann

\footnotetext{
${ }^{a}$ Department of Mechanical Engineering, McCormick School of Engineering and Applied Science, Northwestern University, Evanston, Illinois 60208, USA

${ }^{b}$ Max Planck Institute of Colloids and Interfaces, Science Park Golm, 14424 Potsdam, Germany. E-mail: Rumiana.Dimova@mpikg.mpg.de ${ }^{c}$ Department of Chemistry, Gettysburg College, Gettysburg, Pennsylvania 17325, USA

${ }^{d}$ Department of Engineering Sciences and Applied Mathematics, McCormick School of Engineering and Applied Science, Northwestern University, Evanston,

Illinois 60208, USA. E-mail: petia.vlahovska@northwestern.edu
}

equation $^{9-19}$ (reviews on the subject can be found in ref. 20-22). These models, which are strictly valid only for systems with monovalent charges, do predict an increase in the bending rigidity with membrane surface charge.

Experimental data, albeit limited, ${ }^{22}$ in general support the theoretical results. Of particular interest is the variation of the bending rigidity with surface charge, however, this problem has been investigated either only for relatively low fractions of the ionic species (lipids or surfactants) ${ }^{23-28}$ or on purely ionic lyotropic systems. ${ }^{29,30}$ Giant unilamellar vesicles (GUVs) represent a suitable system for systematic measurements on membranes with good control of the composition. ${ }^{31-34}$ Other advantages of using GUVs are that membranes made of biologically relevant lipids can be studied, and potentially complicating effects due to either high curvature in submicron-sized liposomes or interactions with other bilayers in lamellar stacks are absent. The first attempt to measure the bending rigidity of charged membranes using GUVs was conducted by Song and Waugh ${ }^{25}$ by tether formation. Membranes made of 1-stearoyl-2-oleoyl-snglycero-3-phosphatidylcholine (SOPC) and 1-palmitoyl-2-oleoylsn-glycero-3-phosphatidyl-L-serine (sodium salt) (POPS) show negligible stiffening for up to a $16 \%$ POPS molar fraction. Using fluctuation spectroscopy of giant unilamellar vesicles, Rowat et al. ${ }^{26}$ detected a modest increase of $3-5 k_{\mathrm{B}} T$ in the bending rigidity for a membrane system of dimyristoyl phosphatidylcholine with 1-5 mol\% ionic surfactants, and Mitkova et $a{ }^{27}$ measured an increase of $7 k_{\mathrm{B}} T$ for a $20 \%$ fraction of negatively charged lipids in membranes made of SOPC:DOPS (dioleolylphosphatidylcholine) in acidic solutions. Membranes containing larger fractions of charged lipids (up to $40 \%$ ) were 
studied by Vitkova et $a .^{28}$ Micropipette aspiration of giant vesicles made of POPC:POPS mixtures showed an increase in membrane bending rigidity by $10 k_{\mathrm{B}} T$. However, these experiments were conducted in a high sucrose concentration ( $0.17 \mathrm{M}$ sucrose), at which sugars bind quite strongly to a lipid bilayer, resulting in membrane lateral expansion and thinning. ${ }^{35}$ This results in a softer membrane, ${ }^{36,37}$ making it difficult to isolate the electrostatic contribution to the membrane bending rigidity. Overall, a comprehensive investigation of the bending rigidity as a function of surface charge over a broad range of charged lipid fraction is lacking.

In this paper, we report an experimental study of the dependence of the bending rigidity on the charge content of bilayer membranes using GUVs made of a mixture of neutral and monovalently charged lipids. The whole range of $0-100 \%$ lipid charge fraction is explored. We take precautions to minimize the effects of sugars, salt and bilayer asymmetry. We implement two independent non invasive techniques, equilibrium shape fluctuations and time correlations, to measure the membrane bending rigidity. Several new statistical methods are introduced to improve the equilibrium shape fluctuation analysis. The effect of surface charge is also investigated at different ionic strengths of the suspending solution. The experimental results are compared to existing theoretical models of the bending rigidity of membrane systems with monovalent charges.

\section{Materials and methods}

\subsection{Materials}

Giant unilamellar vesicles (GUVs) were prepared from lipids, 1-palmitoyl-2-oleoyl-sn-glycero-3-phosphatidylcholine (POPC) and 1-palmitoyl-2-oleoyl-sn-glycero-3-phosphatidyl-(1'-rac-glycerol) (sodium salt) (POPG), using the electroformation ${ }^{38,39}$ or spontaneous swelling method; ${ }^{40,41}$ see Dimova and Marques ${ }^{34}$ for an overview of the preparation methods. The lipids were purchased from Avanti Polar Lipids (Alabaster, AL). The vesicles were fluorescently labeled with 0.05 mol\% of 1,2-dioleoyl-sn-glycero3-phosphatidylethanolamine- $N$-(lissamine rhodamine B sulfonyl) (ammonium salt), 18:1 Liss Rhod PE (Avanti), to detect defects. All other chemicals were purchased from Sigma unless stated otherwise.

\subsection{Vesicle preparation}

Stock solutions of POPC and POPG at $10 \mathrm{mg} \mathrm{ml}^{-1}$ in chloroform were mixed at different molar ratios to a final concentration of $5 \mathrm{mM}$. For electroformation, a small volume $(5 \mu \mathrm{l})$ of lipid solution was spread on the conductive surface of two glass slides coated with indium tin oxide (ITO) (Delta Technologies). The glass slides were then stored under a vacuum for 1-2 hours to remove traces of organic solvent. Afterwards, a $2 \mathrm{~mm}$ Teflon spacer was sandwiched between the glass slides and the chamber was gently filled with $20 \mathrm{mM}$ sucrose solution or $20 \mathrm{mM}$ sucrose solution with $1 \mathrm{mM} \mathrm{NaCl}$ or $5 \mathrm{mM} \mathrm{NaCl}$. Ultrapure water (22934 HPLC grade, purchased from Fisher Scientific) was used to prepare the sugar/salt suspending solutions.
The slides (conductive side facing inward) were connected to an AC signal generator Agilent 33220A (Agilent Technology $\mathrm{GmbH}$, Germany). An AC field of voltage $1.5 \mathrm{~V}$ and frequency $10 \mathrm{~Hz}$ (for solutions without salt) and $50 \mathrm{~Hz}$ (for solutions with salt) was applied for 2 hours at room temperature, resulting in 10-50 $\mu \mathrm{m}$ sized vesicles. They were stored in amber vials at $25{ }^{\circ} \mathrm{C}$ and were analyzed within a day of formation.

The spontaneous swelling method was also used, especially for GUVs made solely of the charged lipid POPG, which are difficult to electroform. Initially, $30 \mu \mathrm{l}$ of $5 \mathrm{mM}$ (in chloroform) lipid solution was dissolved in $200 \mu \mathrm{l}$ of chloroform in a $20 \mathrm{ml}$ vial. Lipid films were formed from evaporation by blowing a $\mathrm{N}_{2}$ stream. Afterwards, the vials were dried under a vacuum for 1-2 hours. The lipid films were hydrated in the suspending solutions ( $20 \mathrm{mM}$ sucrose solution in $1 \mathrm{mM} \mathrm{NaCl}$ ) and placed at $60{ }^{\circ} \mathrm{C}$ in an oven for 7-8 hours.

After formation, the vesicles were diluted 10 times in $22 \mathrm{mM}$ glucose and $\mathrm{NaCl}$ solution (with the same ionic strength) to achieve better optical contrast for vesicle contour detection.

Charged GUVs prepared via electroformation exhibit transbilayer asymmetry right after electroformation but equilibrate after about 24 hours. ${ }^{42}$ Hence, in order to achieve symmetric leaflets, we analyzed the vesicles 24 hours after electroformation to confirm the absence of tubular structures (tubes are indicative of bilayer asymmetry). We also worked with vesicles under symmetric salt solutions (same concentration inside and outside) to avoid effects associated with membrane curvature. ${ }^{43}$

To quantify the surface charge in the POPC/POPG membranes, we measured the zeta potential of large unilamellar vesicles (LUVs). ${ }^{44}$ LUVs were prepared with the extrusion method. Lipid films were evaporated with a stream of $\mathrm{N}_{2}$ from $5 \mathrm{mg} \mathrm{ml}^{-1}$ stock solutions of POPC and POPG in chloroform and then dried under a vacuum for two hours to remove any trace of organic solvent. After that the lipid films were hydrated in their respective aqueous solution (20 mM sucrose with various concentrations of $\mathrm{NaCl}$ ) to a final lipid concentration of $2.75 \mathrm{mM}$ via 10 minutes of vortexing at room temperature. The vesicles were treated with 7 freeze/thaw cycles in ethanol/dry ice and warm water baths and then extruded 11 times through a $100 \mathrm{~nm}$ pore polycarbonate filter (Avanti).

\subsection{Measurement of the bending rigidity}

We employ the fluctuation spectroscopy technique, which analyses the thermal undulations of a membrane. The details of the procedure are given in ref. 45 . In essence, a time series of vesicle contours in the focal plane (the equator of the quasi-spherical vesicle) is recorded. The quasi-circular contour is decomposed into Fourier modes, $r(\phi)=R\left(1+\sum_{q} u_{q}(t) \exp (i q \phi)\right)$. The fluctuating amplitudes $u_{q}$ are independent and have a mean square amplitude dependent on the membrane bending rigidity $\kappa$ and the tension $\sigma$

$$
\left\langle\left|u_{q}\right|^{2}\right\rangle=\frac{k_{\mathrm{B}} T}{\kappa\left(q^{4}+\bar{\sigma} q^{2}\right)}
$$


where $k_{\mathrm{B}} T$ is the thermal energy $\left(k_{\mathrm{B}}\right.$ is the Boltzmann constant and $T$ is the temperature), and $\bar{\sigma}=\sigma R^{2} / \kappa$.

The equilibrium shape fluctuations are stable and relax with rate $^{46,47}$

$$
\gamma_{q}=\frac{\kappa\left(q^{3}+\bar{\sigma} q\right)}{4 \mu R^{3}}
$$

which appears in the time autocorrelation function

$$
C(q, \Delta t)=\left\langle u_{q}(t) u_{-q}(t+\Delta t)\right\rangle=\left\langle\left|u_{q}\right|^{2}\right\rangle \exp \left(-t \gamma_{q}\right)
$$

The time autocorrelation function is normalized by $C(q, \Delta t=0)$ to isolate the effect of the relaxation rates.

$$
C_{\text {norm }}=\frac{C(q, \Delta t)}{C(q, \Delta t=0)}
$$

Note that eqn (2) assumes that the suspending solution and the solution enclosed by the vesicle have the same viscosity $\mu$, which is approximately the case for our system.

In the experiment, we check that the membrane fluctuation modes are independent by computing the temporal cross correlations

$$
G(\Delta t)=\left\langle u_{q}(t) u_{q+m}(t+\Delta t)\right\rangle
$$

where $m \neq q$ ( $m=q$ represents the autocorrelation function).

Furthermore, to rule out any effects of external environmental conditions on the fluctuations, we examine if the shape fluctuations $u_{q}$ follow a Gaussian distribution by performing a chi-squared distribution test of every mode number. ${ }^{48}$ This helps to eliminate vesicles that are close to the cover glass where fluctuations can be modified by nearby small structures (SUVs, LUVs, small GUVs or lipid aggregates) below the optical resolution of the microscope that affect the vesicle fluctuations. We chose only vesicles that passed this test with a confidence interval of $95 \%$.

\subsection{Image acquisition and processing}

Membrane fluctuations were observed using a phase contrast and epifluorescent microscope (A1 Axio Observer, Zeiss, Germany) with $100 \times(1.4 \mathrm{NA})$ and $63 \times(0.75 \mathrm{NA})$ (Zeiss, Germany) objectives. High speed video recording and imaging were performed using a SA1.1 high speed camera (Photron, Japan) and Pco.Edge (PCO AG, Kelheim, Germany). Images were acquired at $60 \mathrm{fps}$ but the frame rate was optimized from vesicle to vesicle for equilibrium fluctuation analysis to ensure image acquisition slower than the mode correlation time eqn (2); typical rates ranged from 10 to $20 \mathrm{fps}$. For time correlations, the image acquisition rate was set to $1000 \mathrm{fps}$, in order to resolve the mode dynamics on the time scale eqn (2).

In order to reduce the influence of the integration time effect, a shutter speed was used corresponding to an exposure time of $200 \mu$ s. 10000 images were obtained for each set of experiments. Only defect-free quasi-spherical vesicles were analyzed.

We developed our own software for vesicle contour detection. The program was coded and compiled in MATLAB (Mathworks, USA) with the help of in-built MATLAB functions. The software performs three important steps to detect the contour: (i) image processing, (ii) pixel and sub-pixel resolution contour detection and (iii) fitting the vesicle contour by a Fourier series.

Based on the resolution of the microscope and the high speed camera, for a vesicle $20 \mu \mathrm{m}$ in radius, the number of resolved modes is approximately 20 . The fluctuation spectrum is sensitive to the bending rigidity only for modes larger than $q_{\mathrm{c}}=R \sqrt{\sigma / \kappa}\left(q<q_{\mathrm{c}}\right.$ modes are governed by tension). Hence, we worked with vesicles with low tension values $10^{-7}-10^{-9} \mathrm{~N} \mathrm{~m}^{-1}$ and radius size $10-20 \mu \mathrm{m}$ to ensure low crossover modes $q_{\mathrm{c}} \sim 3-7$.

\subsection{Dynamic light scattering and zeta potential measurements}

Zeta potential measurements with GUVs are challenging, hence we used LUVs since their composition and zeta potential correlate well with those of GUVs. ${ }^{44}$ The zeta potential and vesicle diameter were measured at $22{ }^{\circ} \mathrm{C}$ by quasi-elastic light scattering analysis with a Zetasizer ZS90 (Malvern Instruments, Worcestershire, UK) with a HeNe laser $(632.8 \mathrm{~nm})$, a scattering angle of $90^{\circ}$, and a temperature controlled cuvette holder. The vesicle size was determined with five dynamic light scattering measurements consisting of 10-20 consecutive runs to obtain intensity size distributions. The diameter of the LUVs formed from the extrusion process for different suspending solutions was in the range $110-170 \mathrm{~nm}$. For the zeta potential, $2.75 \mathrm{mM}$ lipid concentration samples were run in DST1070 folded capillary cells with integral gold electrodes (Malvern). The zeta potential was deduced from the electrophoretic mobility, $\mu_{\mathrm{e}}$, data using the Henry equation:

$$
\zeta=\left(3 \mu_{\mathrm{e}} \mu\right) /\left(2 \varepsilon_{\mathrm{w}} \varepsilon f\left(R / \lambda_{\mathrm{D}}\right)\right)
$$

where $\mu$ is the viscosity of the aqueous solution, $\lambda_{\mathrm{D}}$ is the Debye length, $\varepsilon$ and $\varepsilon_{\mathrm{w}}$ are the permittivity of free space and the relative permittivity of the medium, and the Henry function, $f\left(R / \lambda_{\mathrm{D}}\right)$, depends on the radius $R$ of the vesicle and the Debye length thickness. The Huckel approximation $f\left(R / \lambda_{\mathrm{D}}\right)=1$ applies for small particles with a relatively thick double layer, such as when $R / \lambda_{\mathrm{D}}<1$, while the Smoluchowski approximation $f\left(R / \lambda_{\mathrm{D}}\right)=1.5$ applies to large particles with a relatively thin double layer, $R / \lambda_{\mathrm{D}}>1000$. An intermediate prefactor, $f\left(R / \lambda_{\mathrm{D}}\right)=1 / 6 \log \left(R / \lambda_{\mathrm{D}}\right)+1$, is used when $1<R / \lambda_{\mathrm{D}}<1000$; in this case, for all vesicle solutions containing $\mathrm{NaCl}$ (1-100 mM). This dependence was taken into account in the analysis of the zeta potential measurements. ${ }^{49}$

\section{Results and discussion}

Fig. 1 shows a typical spectrum of the shape fluctuations and illustrates the $q^{-4}$ dependence of the mean-squared amplitude on the mode number. For this measurement it is important that the modes are equilibrated, i.e., the acquisition rate (data sampling) is slower than the correlation time of the modes (given by eqn (2)). As seen in Fig. 2a, which plots the time autocorrelation function of modes $q=2 \ldots 7$, all modes above wavenumber 3 are equilibrated after about $0.1 \mathrm{~s}$, suggesting an image acquisition rate of $10 \mathrm{fps}$. Fitting the data in Fig. 1 with eqn (1) yields $\kappa=42.1 \pm 3.0 k_{\mathrm{B}} T$ and $\sigma=7 \pm 2 \times 10^{-9} \mathrm{~N} \mathrm{~m}^{-1}$. 


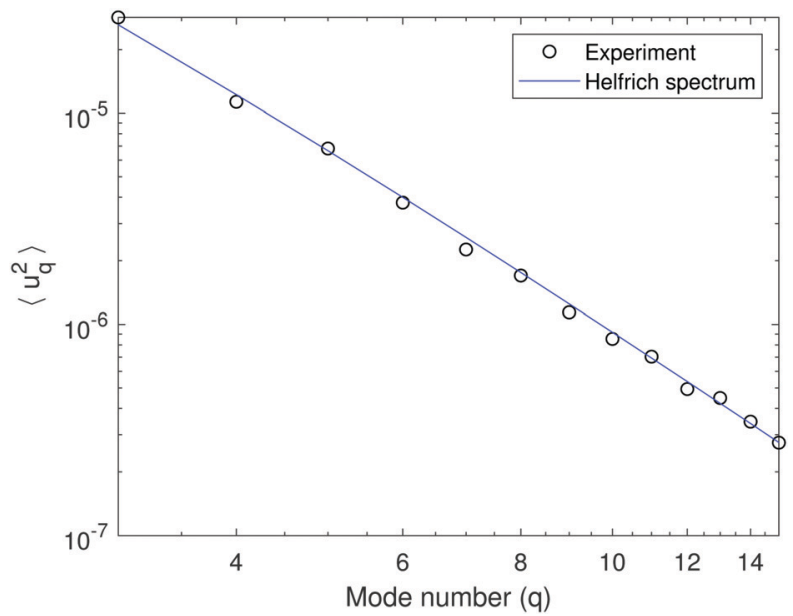

Fig. 1 A fluctuation spectrum of a 50:50 POPC:POPG GUV in the absence of salt. The variance of the amplitude $\left\langle\left|u_{q}\right|^{2}\right\rangle$ dependence on mode number $q$ if fitted with eqn (1) yields bending rigidity $\kappa=42.1 \pm 3.0 \mathrm{k}_{\mathrm{B}} T$ and tension $\sigma=7 \pm 2 \times 10^{-9} \mathrm{~N} \mathrm{~m}^{-1}$. The vesicle radius is $R=12.7 \mu \mathrm{m}$.
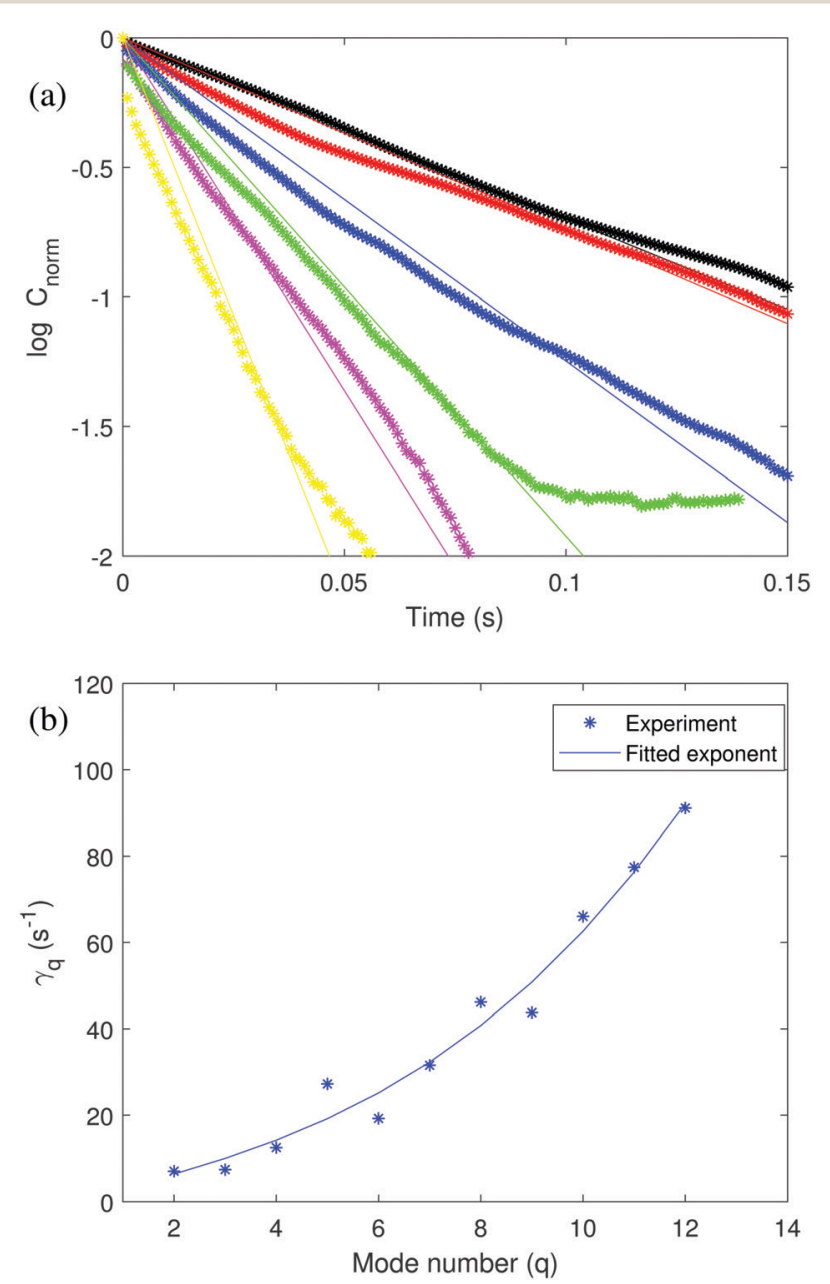

Fig. 2 (a) Normalized time autocorrelation function of a 50:50 symmetric POPC : POPG GUV (same vesicle as in Fig. 1) for mode number $2 \leq$ $q \leq 7$. Black, red, blue, green, magenta and yellow symbols represent $q$ from 2 to 7 in order. The solid lines correspond to the fit to the experimental data with theoretical eqn (4). (b) Relaxation dynamics of the shape fluctuations of a $50: 50$ symmetric POPC: POPG GUV. The fitted eqn (2) yields the bending rigidity $\kappa=49.1 \pm 6.4 k_{\mathrm{B}} T$.
A higher acquisition rate, $50 \mathrm{fps}$, results in a higher bending rigidity $\kappa=45.3 \pm 2.5 k_{\mathrm{B}} T$ due to the mode correlations. Such correlations are observed for all examined compositions.

The time autocorrelation data can also be used to extract the bending rigidity. The decay rates extracted from the slopes in Fig. 2 (top panel) were fitted with eqn (2) in Fig. 2 (bottom panel). The obtained value of the bending rigidity is $\kappa=49.1 \pm$ $6.4 k_{\mathrm{B}} T$, a value somewhat higher than the one determined from the fluctuation spectrum. The discrepancy likely originates from the more noisy time correlation data.

\subsection{Membrane stiffening at increased surface charge}

We measured the bending rigidity of membranes with composition ranging from a $100 \%$ neutral lipid, POPC, to a $100 \%$ charged lipid, POPG, in $20 \mathrm{mM}$ sucrose in the absence of salt, and in the presence of $1 \mathrm{mM}$ and $5 \mathrm{mM} \mathrm{NaCl}$. Between 18 to 20 vesicles were analyzed for each composition unless stated otherwise.

The bending rigidity determined from shape fluctuation analysis for different vesicle compositions is shown in Fig. 3 for the system without added salt. The data show that surface charge significantly stiffens the membrane: adding just $20 \%$ of charged lipids increases the bending rigidity by as much as $80 \%$ relative to the charge-free membrane (from $28 k_{\mathrm{B}} T$ to about $50 k_{\mathrm{B}} T$ ).

The addition of salt screens the surface charge thereby decreasing the electrostatic contribution to the bending rigidity. Fig. 4 and 5 confirm that the membrane stiffening with charged lipid fraction is a lot less pronounced in $1 \mathrm{mM}$ and $5 \mathrm{mM} \mathrm{NaCl}$ salt. Note that we have plotted data only from the equilibrium fluctuation spectrum in Fig. 4 and 5 for the saltcontaining cases. We found it experimentally challenging to grow large charged GUVs $(R>10 \mu \mathrm{m})$ in salt-containing solutions. This affected the number of modes resolved from the time autocorrelation function.

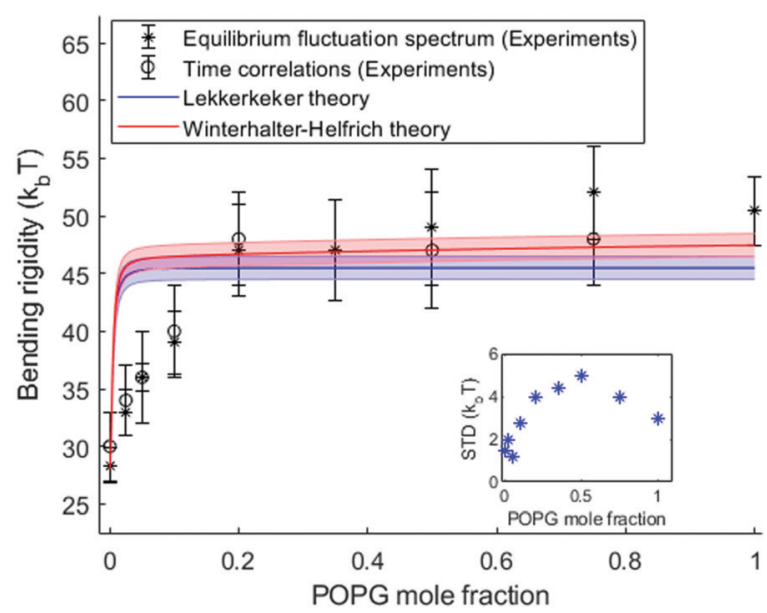

Fig. 3 Bending rigidity as a function of the fraction of charged lipids in the absence of added salt. The blue and red solid lines represent the theoretical predictions by the Lekkerkerker and Winterhalter and Helfrich models eqn (8). The color bands represent the uncertainty introduced in the theoretical predictions by solution conductivity measurements. The inset shows the standard deviation in the bending rigidity as a function of the amount of charged lipids. 


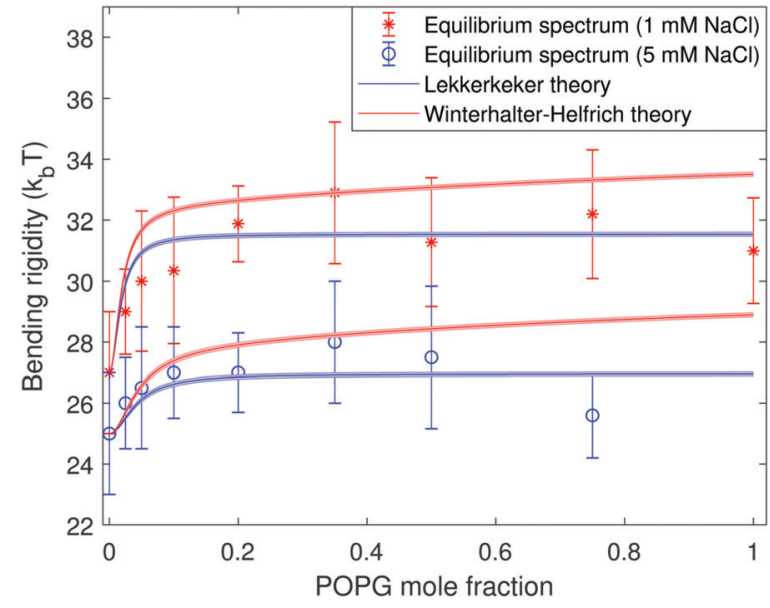

Fig. 4 Bending rigidity as a function of the fraction of charged lipids in the membrane in the presence of $1 \mathrm{mM} \mathrm{NaCl}$ (red stars) and $5 \mathrm{mM} \mathrm{NaCl}$ (blue circles). The blue and red solid lines represent the Lekkerkerker and the Winterhalter-Helfrich theories eqn (8), respectively. The color bands represent the uncertainty introduced in the theoretical predictions by the solution conductivity measurements.

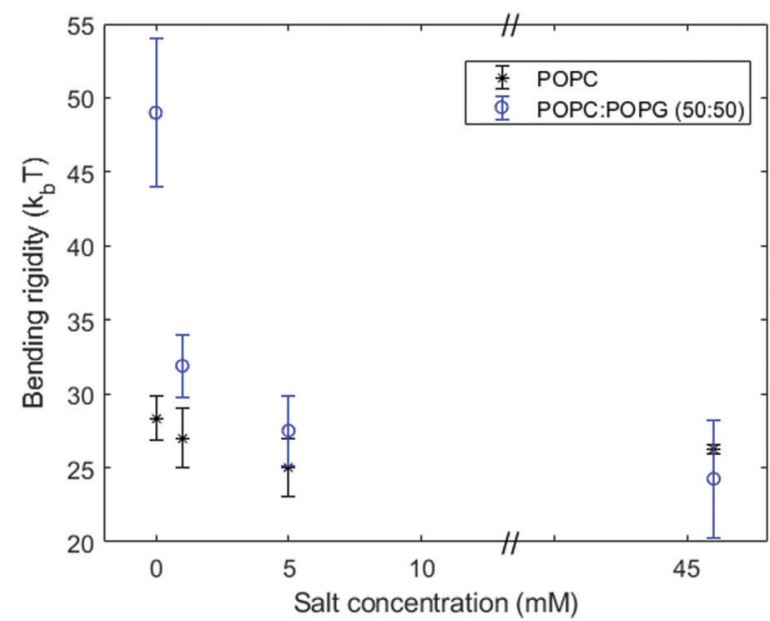

Fig. 5 Bending rigidity (from the equilibrium fluctuation spectrum) of pure POPC and POPC:POPG $(50: 50)$ membranes as a function of the $\mathrm{NaCl}$ concentration in the bulk solution.

Several theoretical models have been developed for the membrane stiffening due to surface charge $\mathrm{e}^{9-12,14,15,17-19}$

$$
\kappa=\kappa_{0}+\kappa_{\mathrm{el}}
$$

where $\kappa_{0}$ is the rigidity of the neutral membrane (in our case POPC) and $\kappa_{\mathrm{el}}$ is the electrostatic contribution due to surface charge. We compared our experimental data with the theoretical predictions for the electric contribution to the bending rigidity derived by Lekkerkerker ${ }^{11}\left(\kappa^{\mathrm{el}, \mathrm{L}}\right)$ and Winterhalter and Helfrich $^{15}\left(\kappa^{\mathrm{el}, \mathrm{WH}}\right)$. The Lekkerkerker ${ }^{11}$ model solves the nonlinear Poisson-Boltzmann equation to get the electrostatic energy of a charged spherical vesicle. Winterhalter and Helfrich $^{15}\left(\kappa^{\mathrm{el}, \mathrm{WH}}\right)$ modified Lekkerkerker's theory $\left(\kappa^{\mathrm{el}, \mathrm{L}}\right)$ by accounting for the fact that the neutral surface of each monolayer may not directly coincide with the position of the head group charges. The theoretical equations are:

$$
\begin{aligned}
\kappa^{\mathrm{el}, \mathrm{L}}= & \frac{\lambda_{\mathrm{D}} k_{\mathrm{B}} T}{Q \pi} \frac{q_{\mathrm{e}}-1}{q_{\mathrm{e}}} \frac{\left(q_{\mathrm{e}}+2\right)}{\left(q_{\mathrm{e}}+1\right)} \\
\kappa^{\mathrm{el}, \mathrm{WH}}= & \kappa^{\mathrm{el}, \mathrm{L}}+\frac{\lambda_{\mathrm{D}} k_{\mathrm{B}} T}{Q \pi} \frac{q_{\mathrm{e}}-1}{q_{\mathrm{e}}} \\
& \times\left[4 \lambda_{\mathrm{D}}{ }^{-1}(\delta-\eta)+2 \lambda_{\mathrm{D}}{ }^{-2}\left(q_{\mathrm{e}}+1\right)(\delta-\eta)^{2}\right]
\end{aligned}
$$

where $\lambda_{\mathrm{D}}$ is the Debye length, $\delta$ is the bilayer thickness, $\eta$ is the distance between the monolayer neutral surface and the mid-plane of the bilayer, $Q=\frac{e^{2}}{4 \pi \varepsilon \varepsilon_{\mathrm{W}} k_{\mathrm{B}} T}$ is the Bjerrum length, $e$ is the electronic charge, and $q_{\mathrm{e}}=\sqrt{p^{2}+1}$, where $p=$ $\left(2 \pi Q \Lambda \lambda_{\mathrm{D}}\right) / A$, where $\Lambda$ is the fraction of charged head groups and $A$ is the area per molecule. The theories, which involve no adjustable parameters, show relatively good agreement with the experiments, see Fig. 3 . The model parameters $\left(\delta, \eta, \lambda_{\mathrm{D}}, Q, A\right)$ for the theoretical curves were experimentally determined. $Q$ is $0.714 \mathrm{~nm}$ for a $1: 1$ electrolyte solution at $25{ }^{\circ} \mathrm{C}$. The POPC and POPG area per lipid considered are $68.3 \mathrm{~A}^{2}$ and $66.1 \mathrm{~A}^{2}$ respectively. ${ }^{50,51}$ For intermediate POPG fractions, we assumed a linear change in the area per lipid with varying POPG molar fraction. At room temperature, the bilayer thickness (the monolayer thickness, $\delta$, is $1 / 2$ of the bilayer thickness) and the hydrocarbon region thickness (the hydrophobic tail thickness, $\eta$, is $1 / 2$ of the hydrocarbon region thickness) for POPC are $3.91 \mathrm{~nm}$ and $2.88 \mathrm{~nm}$ respectively. ${ }^{51,52}$ Similarly, the bilayer thickness and the hydrocarbon region thickness of POPG are $3.66 \mathrm{~nm}$ and $2.78 \mathrm{~nm}$ respectively. ${ }^{51,52}$ Linear approximations of the thickness values were made for intermediate compositions with varying POPG molar content. The Debye length was calculated from the measured solution conductivity

$$
\lambda_{\mathrm{D}}=\sqrt{\frac{\varepsilon_{0} \varepsilon_{\mathrm{w}} D}{K_{\mathrm{c}}}}
$$

where $D$ is the diffusivity of charge carriers (ions) in the bulk solution. At room temperature, $298 \mathrm{~K}$, using a typical value for the ion diffusion constant given by the average of the $\mathrm{Na}^{+}$and $\mathrm{Cl}^{-}$ions, $D=1.7 \mu \mathrm{m}^{2} \mathrm{~ms}^{-1}$, and for the dielectric constant of water, $\varepsilon_{\mathrm{w}}=$ $78.54,{ }^{53}$ yields a Debye length of $38.2 \pm 2.2 \mathrm{~nm}$ for the salt-free solution with measured conductivity $K_{\mathrm{c}}=8 \pm 1 \mu \mathrm{S} \mathrm{cm}^{-1}$. The Debye lengths for the $1 \mathrm{mM}$ and $5 \mathrm{mM} \mathrm{NaCl}$ solutions estimated from the measured conductivities $K_{\mathrm{c}}=126 \pm 1 \mu \mathrm{S} \mathrm{cm}^{-1}$ and $K_{\mathrm{c}}=617 \pm$ $6 \mu \mathrm{S} \mathrm{cm} \mathrm{cm}^{-1}$ are $\lambda_{\mathrm{D}}=9.67 \pm 0.03 \mathrm{~nm}$ and $\lambda_{\mathrm{D}}=4.37 \pm 0.02 \mathrm{~nm}$, respectively. These values are essentially the same as those calculated from the standard formula using the monovalent salt concentration $c$ in $\mathrm{M}, \lambda_{\mathrm{D}}=0.304 / \sqrt{c} \mathrm{~nm}$ at room temperature $25{ }^{\circ} \mathrm{C}$.

The agreement between theory and experimental data is very good in the salt-containing systems as seen in Fig. 4. In both the salt-free and salt-containing solutions the agreement between the experimental data and the Lekkerkerker model is better compared to the more complex Winterhalter and Helfrich one. In the case of the salt-free solutions, both models overestimate the membrane rigidity at low surface charge, see 
Fig. 3. This may be due to the approximations made in the model, such as the assumption of spherical geometry. On the experimental side, impurities in the commercially obtained chemicals $^{4,54}$ or variations in membrane composition due to preparation issues ${ }^{42,55}$ could lead to underestimation of the surface charge. Discrepancies between the starting lipid mixture and the final vesicle composition are suggested by the observed behavior of the standard deviation in the measured bending rigidity (from the equilibrium fluctuation spectrum), see the inset in Fig. 3: it increases from a purely neutral POPC membrane $\left(1.5 k_{\mathrm{B}} T\right)$ to a mixed $50: 50$ composition $\left(5 k_{\mathrm{B}} T\right)$ and decreases again for a fully charged POPG membrane $\left(3 k_{\mathrm{B}} T\right)$. To check if the preparation protocol influences the results, we compared the bending rigidity of GUVs with $10 \%$ of POPG

(a)

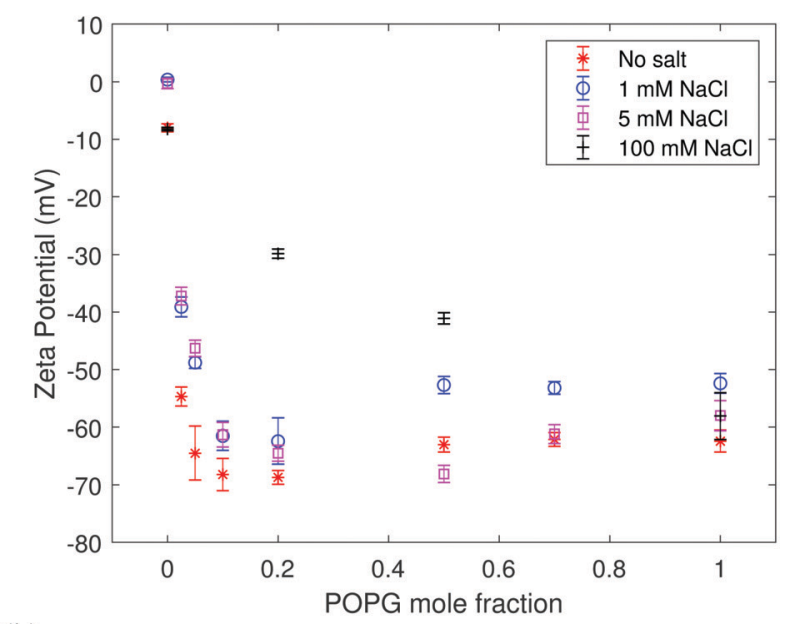

(b)

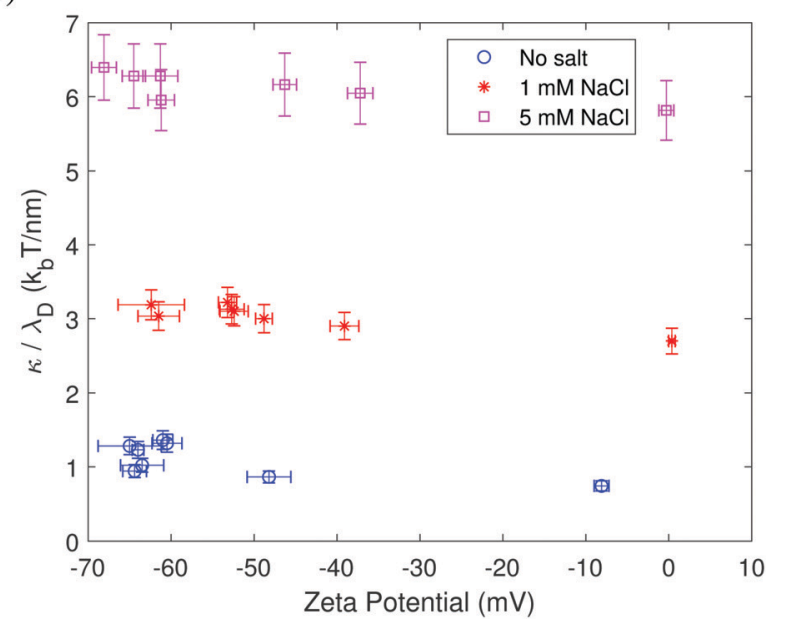

Fig. 6 (a) Zeta potential measured for extruded POPC:POPG liposomes prepared in $20 \mathrm{mM}$ sucrose solution and different salt conditions. The standard deviation is calculated over average zeta potential values for six different experiments for each lipid composition. (b) Bending rigidity (from the equilibrium fluctuation spectrum) scaled by the Debye length as a function of zeta potential. The results show that the bending rigidity of membranes of arbitrary surface charge can be estimated from that of the neutral membrane measured at the respective salt concentration after rescaling by the respective Debye length. prepared by electroformation and natural swelling and found them to be virtually the same, $39.01 \pm 4.00 k_{\mathrm{B}} T$ and $37.75 \pm$ $2.87 k_{\mathrm{B}} T$, correspondingly. This indicates that the discrepancy with the theory at low surface charge density and no added salt does not originate from the experimental protocol.

\subsection{Zeta potential and bending rigidity}

The dependence of the zeta potential on surface charge shown in Fig. 6 mirrors the bending rigidity trend seen in Fig. 3-5. Fig. 6 a shows that a higher proportion of charged lipids results in a more negative zeta potential value. The screening effect due to the addition of salt is also evident: in the absence of salt, the zeta potential is more negative than in the presence of $1 \mathrm{mM} \mathrm{NaCl}$ and $5 \mathrm{mM} \mathrm{NaCl}$. The screening effect becomes very pronounced at a high salt concentration of $100 \mathrm{mM} \mathrm{NaCl}$. Fig. 6b shows that the ratio of the bending rigidity and Debye length is insensitive to the zeta potential. This behavior is predicted by Lekkerkerker's theoretical model eqn (8) in the limit of high surface charge $p \approx q \gg 1, \kappa^{\mathrm{el}, \mathrm{L}} / \lambda_{\mathrm{D}} \sim k_{\mathrm{B}} T / Q \pi$. These results confirm the trend seen in Fig. 3 and 4 that the bending rigidity quickly becomes insensitive to the charged lipid fraction above a few \% POPG. The results in Fig. $6 \mathrm{~b}$ also imply that the bending rigidity of membranes with an increasing fraction of charged lipids can be directly assessed from that of the neutral membrane measured at the respective salt concentration after rescaling by the respective Debye length.

\section{Conclusions and outlook}

We experimentally studied the effects of surface charge on the bending rigidity of lipid bilayer membranes. Using POPC (neutral)/ POPG (negatively charged) lipids we quantifed the membrane stiffening in the full range of membrane compositions - from neutral to $100 \%$ charged - as well as at different salt concentrations. To measure the bending rigidity we employed the fluctuation spectroscopy technique with giant unilamellar vesicles. We analyzed both the mean amplitude and the time autocorrelations of the shape fluctuation modes. Our results show that the membrane bending rigidity increases rapidly with surface charge and for the first time demonstrate the theoretically predicted plateau above $20 \%$ surface charge. Addition of salt to the bulk solutions reduces the bending rigidity of the charged membranes due to surface charge screening. The experimental data are in very good agreement with the theoretical models, without any adjustable parameters. Only for intermediate fractions of the charged lipid and in the absence of salt do the theoretical models overestimate the bending rigidity, suggesting the need for further improvement either of the theoretical models or the experimental precision. The trends in bending rigidity qualitatively match the dependence of the zeta potential of LUVs on the membrane composition and bulk salt concentration.

Our contribution highlights the importance of charge in membrane mechanics. A question that still remains to be addressed experimentally relates to the effect of multivalent 
ions such as calcium and magnesium on the bending rigidity of membranes. Multivalent ions are theoretically predicted to soften the membrane, ${ }^{56-58}$ and experimental data are scarce. ${ }^{59}$ Our experimental methodology using GUV shape fluctuations provides a foundation for future studies on membrane electromechanics such as the effect of multivalent ions on the membrane dynamics and elastic properties.

\section{Conflicts of interest}

There are no conflicts to declare.

\section{Acknowledgements}

This research was funded in part by NSF-CMMI awards 1748049 and 1740011. PV acknowledges support from the Alexander von Humboldt Foundation for a visit to the Max Planck Institute of Colloids and Interfaces. JS and RD acknowledge the stimulating environment of the MaxSynBio consortium which is jointly funded by the Federal Ministry of Education and Research in Germany and the Max Planck Society. Open Access funding provided by the Max Planck Society.

\section{References}

1 L. Chernomordik and M. Kozlov, Nat. Struct. Mol. Biol., 2008, 15, 675-683.

2 J. C. Stachowiak, F. M. Brodsky and E. A. Miller, Nat. Cell Biol., 2013, 15, 1019-1027.

3 P. Bassereau, R. Jin, T. Baumgart, M. Deserno, R. Dimova, V. A. Frolov, P. V. Bashkirov, H. Grubmüller, R. Jahn, H. J. Risselada, L. Johannes, M. M. Kozlov, R. Lipowsky, T. J. Pucadyil, W. F. Zeno, J. C. Stachowiak, D. Stamou, A. Breuer, L. Lauritsen, C. Simon, C. Sykes, G. A. Voth and T. R. Weikl, J. Phys. D: Appl. Phys., 2018, 51, 343001.

4 R. Dimova, Adv. Colloid Interface Sci., 2014, 208, 225-234.

5 W. Kim, W. Zhu, G. Hendricks, D. Van Tyne, A. Steele, C. Keohane, N. Fricke, A. Conery, S. Shen, W. Pan, K. Lee, R. Rajamuthiah, B. Fuchs, P. Vlahovska, W. Wuest, M. Gilmore, H. Gao, F. Ausubel and E. Mylonakis, Nature, 2018, 556, 103-107.

6 Y.-F. Chen, T.-L. Sun, Y. Sun and H. W. Huang, Biochemistry, 2014, 53, 5384-5392.

7 T.-Y. Lin and D. B. Weibel, Appl. Microbiol. Biotechnol., 2016, 100, 4255-4267.

8 W. Dowhan, Annu. Rev. Biochem., 1997, 66, 199-232.

9 M. Winterhalter and W. Helfrich, J. Phys. Chem., 1988, 92, 6865 .

10 D. J. Mitchell and B. W. Ninham, Langmuir, 1989, 5, 1121.

11 H. N. W. Lekkerkerker, Physica A, 1989, 159, 319-328.

12 H. N. W. Lekkerkerker, Physica A, 1990, 167, 384-394.

13 A. Fogden, D. J. Mitchell and B. W. Ninham, Langmuir, 1990, 6, 159-162.

14 B. Duplantier, R. E. Goldstein, A. I. Pesci and V. RomeroRochin, Phys. Rev. Lett., 1990, 65, 508.
15 M. Winterhalter and W. Helfrich, J. Chem. Phys., 1992, 96, 327-330.

16 J. Harden, C. Marques, J. Jonanny and D. Andelman, Langmuir, 1992, 8, 1170-1175.

17 P. A. Kralchevsky, T. D. Gurkov and K. Nagayama, J. Colloid Interface Sci., 1996, 180, 619-622.

18 S. May, J. Chem. Phys., 1996, 105, 8314-8323.

19 B. Loubet, P. L. Hansen and M. A. Lomholt, Phys. Rev. E: Stat., Nonlinear, Soft Matter Phys., 2013, 88, 062715.

20 D. Andelman, Structure and dynamics of membranes, Elsevier, 1995, pp. 603-641.

21 A. Fogden and B. Ninham, Adv. Colloid Interface Sci., 1999, 83, 85-110.

22 I. Bivas and Y. A. Ermakov, Advances in Planar Lipid Bilayers and Liposomes, 2006, vol. 5, pp. 313-343.

23 R. Bradbury and M. Nagao, Soft Matter, 2016, 12, 9383-9390.

24 R. Schomaecker and R. Strey, J. Phys. Chem., 1994, 98, 3908-3912.

25 J. Song and R. E. Waugh, J. Biomech. Eng., 1990, 112, 235-240.

26 A. C. Rowat, P. L. Hansen and J. H. Ipsen, EPL, 2004, 67, 144.

27 D. Mitkova, N. Marukovich, Y. A. Ermakov and V. Vitkova, Colloids Surf., A, 2014, 460, 71-78.

28 V. Vitkova, J. Genova, O. Finogenova, M. Mitov, Y. Ermakov and I. Bivas, C. R. Acad. Bulg. Sci., 2004, 57, 25-30.

29 N. Delorme, J. F. Bardeau, D. Carriere, M. Dubois, A. Gourbil, H. Mohwald, T. Zemb and A. Fery, J. Phys. Chem., 2007, 111, 2503-2505.

30 B.-S. Lu, S. P. Gupta, M. Belika, R. Podgornik and G. Pabst, Langmuir, 2016, 32, 13546-13555.

31 R. Dimova, S. Aranda, N. Bezlyepkina, V. Nikolov, K. A. Riske and R. Lipowsky, J. Phys.: Condens. Matter, 2006, 18, S1151-S1176.

32 P. Walde, K. Cosentino, H. Engel and P. Stano, ChemBioChem, 2010, 11, 848-865.

33 R. Dimova, Annu. Rev. Biophys., 2019, 48, 93-119.

34 R. E. Dimova and C. E. Marques, The Giant Vesicle Book, CRC Press, Boca Raton, 2020, DOI: 10.1201/9781315152516.

35 H. D. Andersen, C. Wang, L. Arleth, G. H. Peters and P. Westh, Proc. Natl. Acad. Sci. U. S. A., 2011, 108, 1874-1878.

36 V. Vitkova, J. Genova, M. D. Mitov and I. Bivas, Mol. Cryst. Liq. Cryst., 2006, 449, 95-106.

37 D. Mitkova and V. Vitkova, Russ. J. Electrochem., 2016, 52, 1172-1178.

38 M. I. Angelova and D. S. Dimitrov, Faraday Discuss. Chem. Soc., 1986, 81, 303-311.

39 S. L. Veatch, in Electro-Formation and Fluorescence Microscopy of Giant Vesicles With Coexisting Liquid Phases, ed. T. J. McIntosh, Humana Press, Totowa, NJ, 2007, pp. 59-72.

40 J. P. Reeves and R. M. Dowben, J. Cell. Physiol., 1969, 73, 49-60.

41 H. Stein, S. Spindler, N. Bonakdar, C. Wang and V. Sandoghdar, Front. Physiol., 2017, 8, 63.

42 J. Steinkühler, P. De Tillieux, R. L. Knorr, R. Lipowsky and R. Dimova, Sci. Rep., 2018, 8, 11838-11845.

43 M. Karimi, J. Steinkühler, D. Roy, R. Dasgupta, R. Lipowsky and R. Dimova, Nano Lett., 2018, 18, 7816-7821. 
44 K. Carvalho, L. Ramos, C. Roy and C. Picart, Biophys. J., 2008, 95, 4348-4360.

45 R. S. Gracia, N. Bezlyepkina, R. L. Knorr, R. L. Lipowsky and R. Dimova, Soft Matter, 2010, 6, 1472-1482.

46 F. Brochard and J. F. Lennon, J. Phys., 1975, 36, 1035-1047.

47 J. Seiwert and P. M. Vlahovska, Phys. Rev. E: Stat., Nonlinear, Soft Matter Phys., 2012, 87, 022713.

48 J. R. Taylor, An Introduction to Error Analysis: the Study of Uncertainties In Physical Measurements, University Science Books, Sausalito, Calif., 2nd edn, 1997.

49 B. Klasczyk, V. Knecht, R. Lipowsky and R. Dimova, Langmuir, 2010, 26, 18951-18958.

50 N. Kučerka, S. Tristram-Nagle and J. F. Nagle, Biophys. J., 2006, 90, L83-L85.

51 J. Pan, F. A. Heberle, S. Tristram-Nagle, M. Szymanski, M. Koepfinger, J. Katsaras and N. Kučerka, Biochim. Biophys. Acta, Biomembr., 2012, 1818, 2135-2148.
52 N. Kučerka, M.-P. Nieh and J. Katsaras, Biochim. Biophys. Acta, Biomembr., 2011, 1808, 2761-2771.

53 G. Gramse, A. Dols-Perez, M. Edwards, L. Fumagalli and G. Gomila, Biophys. J., 2013, 104, 1257-1262.

54 C. G. Sinn, M. Antonietti and R. Dimova, Colloids Surf., A, 2006, 282-283, 410-419.

55 N. Bezlyepkina, R. Gracia, P. Shchelokovskyy, R. Lipowsky and R. Dimova, Biophys. J., 2013, 104, 1456-1464.

56 A. W. C. Lau and P. Pincus, Phys. Rev. Lett., 1998, 81, 1338-1341.

57 T. T. Nguyen, I. Rouzina and B. I. Shklovskii, Phys. Rev. E: Stat. Phys., Plasmas, Fluids, Relat. Interdiscip. Top., 1999, 60, 7032-7039.

58 Y. Y. Avital, N. Grønbech-Jensen and O. Farago, Eur. Phys. J. E: Soft Matter Biol. Phys., 2014, 37, 69.

59 M. Simunovic, K. Y. C. Lee and P. Bassereau, Soft Matter, 2015, 11, 5030-5036. 